\title{
Lymph-Node-Sampler-Based Nucleic Acid Detection Helps to Diagnose and Control African Swine Fever in Pig Production
}

\section{Xiaowen Li}

College of Veterinary Science and Animal Husbandry: Sam Higginbottom University of Agriculture Technology and Sciences

Yang Li

Shandong New Hope Liuhe Agriculture and Animal Husbandry Technology Co.,Ltd.

\section{Mingyu Fan}

Shandong new hope liuhe agruculture and animal husbandry technology co.,Itd.

\section{Shiran Fan}

Shandong newhope liuhe group co.,Itd.

\section{Wenchao Gao}

Shandong newhope liuhe group co.,Itd.

\section{Peng Yuan}

Shandong newhope liuhe group co.,Itd.

\section{Jing Ren}

Dezhou University

Jingtao Li

Shandong new hope liuhe group co.,Itd.

\section{Jincheng Yu}

Shandong new hope liuhe group co.,Itd.

\section{Weisheng Wu}

Shandong new hope liuhe group co.,Itd.

Junxian Li

Shandong new hope liuhe group co.,Itd.

Qiannan Yu

Shandong new hope liuhe group co.,Itd.

\section{Zhenwen Shao}

Shandong new hope liuhe group co.,Itd.

\section{Zhichun Yan ( $\square$ zhichunyan@vip.tom.com )}

Shandong New Hope Liuhe Co.,Ltd. 
Keywords: African swine fever, lymph node, sampler, real-time PCR, diagnostics

Posted Date: September 14th, 2021

DOI: https://doi.org/10.21203/rs.3.rs-882660/v1

License: (c) (1) This work is licensed under a Creative Commons Attribution 4.0 International License. Read Full License 
1 Lymph-Node-Sampler-Based Nucleic Acid Detection Helps to Diagnose and

2 Control African Swine Fever in Pig Production

3 Running title: Lymph Node Sampler Helps to Diagnose ASF

4 Xiaowen $\mathrm{Li}^{1,2}$, Yang $\mathrm{Li}^{1,2}$, Mingyu Fan ${ }^{1,2}$, Shiran Fan ${ }^{1,2}$, Wenchao Gao ${ }^{1,2}$, Peng Yuan ${ }^{1,2}$,

5 Jing $\mathrm{Ren}^{3}$, Jingtao $\mathrm{Li}^{2}$, Jincheng $\mathrm{Yu}^{2}$, Weisheng $\mathrm{Wu}^{2}$, Junxian $\mathrm{Li}^{2}$, Qiannan $\mathrm{Yu}^{2}$,

6 Zhenwen Shao ${ }^{1,2}$, Zhichun Yan ${ }^{1,2}$

$7{ }^{1}$ Shandong New Hope Liuhe Agriculture and Animal Husbandry Technology Co., Ltd,

8 Dezhou, China

$9 \quad{ }^{2}$ Shandong New Hope Liuhe Group Co., Ltd, Qingdao, China

$10{ }^{3}$ Shandong Provincial Laboratory of Swine Health Big Data and Intelligent Monitoring,

11 Dezhou, China

12 E-mail for correspondence: Dr Zhichun Yan; zhichunyan@vip.tom.com. 


\section{Abstract}

14 Background: African swine fever (ASF) is a highly contagious hemorrhagic and

15 transboundary animal disease. It has rapidly spread to many regions of the world and is

16 responsible for serious production and economic losses. However, clinical diagnosis is

17 impractical for the similar classic symptoms of ASF and several other normal diseases.

18 How to make a definite diagnosis is a top priority.

19 Results: In the present study, lymph node samples were collected by the patented

20 "lymph node sampler", which makes the sampling safer, more efficient and minimally

21 invasive. The ASF virus (ASFV) contents of lymph node sample as well as serum, oral

22 fluid, nasal and rectal swab samples from pig production line were detected by real-

23 time PCR. The big data results demonstrated that the lymph node samples contained

24 more stable and strongly positive ASFV nucleic acid than the porcine exudate and

25 serum samples. Taking the lymph node sample is one of the assistant ways in

26 diagnosing pigs suspected of having ASF. We supervised twenty farms that realized the

27 accurate diagnosis and eliminating of the risk in the shortest time period by using lymph

28 node sampler.

29 Conclusions: Our findings suggest that the lymph node sample is an ideal tissue for

30 diagnosing ASFV infection. The lymph node sampler is a convenient tool for lymph

31 node sampling for practitioners.

32

33 Keywords: African swine fever; lymph node; sampler; real-time PCR; diagnostics 


\section{Background}

African swine fever (ASF) is a devastating swine viral disease that is reportable to the World Organization for Animal Health (OIE). It causes high fever, severe depression, ataxia and hemorrhages in domestic swine and results in a mortality rate approaching $100 \%$ (Ge et al., 2018). ASF is a highly contagious infectious disease and can be transmitted by exposure to infectious pigs or tissues, contaminated swills, transport vehicles, the clothing of animal workers and infected soft ticks (Penrith and Vosloo, 2009; Guinat et al., 2016; Olesen et al., 2018). Airborne transmission has been demonstrated at close range (Wilkinson and Donaldson, 1977; Olesen et al., 2017). Since its emergence in Kenya in the 1920s, ASF remains endemic in sub-Saharan Africa, the Indian Ocean region and eastern Europe (Costard et al., 2009; Oganesyan et al., 2013; Gallardo et al., 2014). In 2007, ASF was confirmed in Georgia and Russia, posing the risk of further dissemination into neighboring countries (Chapman et al., 2011). Since August 2018, ASF has spread to China, the world's largest pork producer and a leading pork importer (Ma et al., 2020). Ongoing outbreaks of ASF afflicted the livestock industry and wiped out $40 \%$ of the nation's pigs, leading to severe socioeconomic losses (Li and Tian, 2018; Zhou et al., 2018; Lu et al., 2020).

The causative agent of the disease, ASF virus (ASFV), belongs to the genus Asfivirus within the Asfarviridae family (Gogin et al., 2013). ASFV is a complex enveloped virus containing a large double-stranded DNA genome that ranges in length from 170 to $193 \mathrm{kbp}$ (Tulman et al., 2009; Dixon et al., 2013). Although scientists 
worldwide have made great efforts to study the etiology and immunology of ASFV, no prophylactic vaccine or treatment options are commercially available due to the complexity of the virions. Fortunately, early detection and eradication are feasible to control the spread of this epidemic disease (Halasa et al., 2016). However, clinical diagnosis is impractical for the similar classic symptoms of ASF and classic swine fever, swine erysipelas and highly pathogenic porcine reproductive and respiratory syndrome. Laboratory diagnosis is indispensable to make a definite diagnosis. OIE recommends two types of laboratory diagnosis, including etiological diagnosis (e.g., polymerase chain reaction, PCR) and serological diagnosis (e.g., enzyme-linked immunosorbent assay). To date, real-time PCR has been widely used to detect ASFV nucleic acids due to its simple, rapid, highly sensitive and specific features. Nevertheless, the quantity and quality of oral, nasal and rectal swabs vary from pig to pig. False-negative results pose a concerning threat to herds. Higher ASFV content was found in blood than in oral, nasal and rectal fluid samples (Olesen et al., 2017; Zhao et al., 2019). However, blood collection is difficult, and stress in pigs cannot be neglected. Moreover, it is highly likely that obtaining blood from pigs carrying viruses will contaminate the sites, increasing the risk of ASFV infection in the herds.

Lymph nodes were collected by dissecting for viral load measurements in many studies (Palya et al., 2018; Senthilkumar et al., 2018; Liu et al., 2019). Slaughter is unavoidable to get the lymph nodes, which is unsafe and complicated. In the present study, a patent lymph node sampler was used to collect lymph node samples in a 
minimally invasive way (Li et al., 2020). The viral loads of lymph nodes as well as oral

fluid, nasal and rectal swab samples from the pig production line were investigated. The application of lymph node samplers in twenty farms shows great potential for the accurate diagnosis and eradication of ASFV.

\section{Materials and methods}

\subsection{The structure and usage of the lymph node sampler.}

The lymph node sampler is a simple and easy-to-operate tool. It consists of a needle, a syringe, a handle and a connection rod inside the needle (Figure 1A, 1B). The needle contains a barb, which can remove the lymphoid tissue from the muscle (Figure 1C, 1D). The connection rod can be inserted into the needle to extrude the tissue.

\subsection{How to use the lymph node sampler.}

First, the pig was restrained to access its inguinal lymph nodes (Figure 2A). Then, the skin was punctured vertically with the sampler to ensure that the barb entirely entered the tissue (Figure 2B). The sampler was pulled out, and the handle was pressed to push the tissue out of the needle (Figure 2C). The tissue was placed into an Eppendorf (EP) tube and submerged in nucleic acid protective fluid.

Plenty of needles should be prepared before the sampling. The sampler can be disassembled for recycling by removing the residual blood and tissue, soaking the sampler for 30 minutes in a sodium hypochlorite solution $(3 \%)$, and washing the sampler with clean water. The sampler can then be reassembled and dried for the next 
usage.

\subsection{Sampling methods of other tissues.}

For serum samples, collect the blood from the porcine anterior vena cava into an anticoagulation tube. Let stand for one hour and get the upper serum. For oral fluid

101 samples, tie a cotton rope in front of the pig. Squeeze out the liquid after the sufficient

102 chew by the pig. For throat swabs, insert a long spermaduct with a sponge on the top 103 into the throat quickly. Elute the mucus with $1 \mathrm{~mL}$ nucleic acid protective fluid (refer to

104 2.4). The cotton nasal swabs and rectal swabs were also eluted with the nucleic acid 105 protective fluid. The nasal-rectal swab and nasal-throat-rectal swab samples were the 106 mixture of the individuals.

\subsection{Preventing sample degradation}

108 The sample should be protected in case of degradation. In this study, a patented

109 customized nucleic acid protective fluid was used, which contains

110 ethylenediaminetetraacetic acid (EDTA) to stop DNase activity by chelating

111 magnesium, manganese and ferric ions (Yu et al., 2017; Ren et al., 2020). The

112 concentrated solution was diluted with purified water. Working solutions of nucleic acid

113 protective fluid normally last for up to 7 days, but fresh solution is recommended.

114 For the lymph node samples, $1 \mathrm{~mL}$ of nucleic acid protective fluid was added to

115 soak the tissue. For the swab samples, the sticks were broken and $1 \mathrm{~mL}$ of nucleic acid

116 protective fluid was added. The samples were sent for detection at room temperature

117 within one day. 


\subsection{Quantitative real-time PCR (qPCR).}

ASFV DNA was quantified with qPCR analysis in a "one step" way without the

120 process of nucleic acid extraction. The kit from Beijing MingRiDa Technology

121 Development Co., Ltd. (010688870) was used according to the user guide. Briefly,

122 lymph node samples were homogenized. Swab samples were oscillated and centrifuged

123 at $8000 \mathrm{~g}$ for $2 \mathrm{~min}$. Five microliters of supernatant, negative control or positive control

124 fluid was mixed with $20 \mu \mathrm{L}$ of operating fluid. Twenty microliters of mixture was taken

125 for qPCR detection.

126

127 3. Results

$128 \quad 3.1$ The ASFV content of lymph nodes was higher than that of other collected

129 sample tissues.

130 Suspected ASF samples from the pig production line in Northwestern Shandong

131 Province from January 2020 to March 2021 were collected for nucleic acid detection

132 by qPCR. The results showed that 37 serum samples, 243 lymph node samples, 237

133 oral fluid, 17 throat swab, 102 nasal swab, 25 rectal swab, 1341 nasal-rectal swab and

13426 nasal-throat-rectal swab samples were true positive for ASFV. The CT values were

135 collected and are shown in Figure 3. Serum samples are widely used for many

136 pathogenic diagnoses. The nasal, nasal-rectal and nasal-throat-rectal swab samples had

137 almost the same CT value as the serum samples, suggesting that the virus contents of

138 nasal and serum samples were comparable. Saliva is other common sampling point. 
139 However, our results showed that the mean CT value of saliva samples was much higher

140 than those of other sites, suggesting that the viral loads in saliva were lower than those

141 in other samples. The lymph node samples were the only samples with lower CT values

142 than the serum samples. The standard deviation (SD) value of the lymph node sample

143 CT value was much smaller than that of serum samples. These data suggest that the

144 ASFV content of lymph node samples is more stable than that of serum samples and is

145 higher than that of other samples.

$146 \quad 3.2$ A larger proportion of strongly positive results was found in lymph node 147 samples.

148 The distribution of CT values between the samples was also analyzed. Generally,

149 CT values over 35 are considered hard to judge, while values less than 30 can be

150 identified as strongly positive results. The percentage of strongly positive lymph node

151 samples was $70.9 \%$, while the relative percentages of strongly positive serum, nasal

152 swab and oral-nasal-rectal swab samples were $45.9 \%, 37.3 \%, 37.4 \%$, respectively

153 (Figure 4). These data suggest that the ASFV content of lymph nodes is much higher

154 than that of other sites, which has great potential for the accurate diagnosis of ASFV.

$155 \quad 3.3$ ASFV-positive pigs were removed within 3 weeks with the help of lymph node

156 samplers.

157 To check whether lymph node samples can help to eliminate ASFV-positive pigs

158 in production, lymph node samplers were immediately applied on farms that tested

159 positive for ASFV nucleic acid. Once abnormal symptoms appeared, such as fever, 
160 inappetence, blood spots, emesis or death, the surrounding samples and porcine lymph

161 node samples were collected and detected for the first time. The positive pigs were

162 removed from the herd according to the biosafety requirements. The surroundings were

163 disinfected repeatedly using 3\% sodium hypochlorite until free from nucleic acid.

164 Among all twenty farms we monitored, the time from ASFV positivity to negativity

165 ranged from 1 day to 18 days (Figure 5). These results demonstrated that lymph node

166 sampling is workful for the removal of ASFV-positive pigs.

\section{4. Discussion}

ASF is an acute viral infection that has a major impact on global pig production.

170 Stockbreeders displayed passivity in the diffusion of practices without effective

171 vaccines or drugs. However, the highly contagious characteristic of ASF transmission

172 indicates the potential for possible interventions to manage the risk of ASF introduction

173 and transmission (Lichoti et al., 2016; Govoeyi et al., 2020). Biosecurity is a high

174 priority for pig farmers due to its high efficiency in controlling this disease (De Lorenzi

175 et al., 2020; Jackman et al., 2020). It contains at least two parts: how to prevent ASF

176 infection entirely and how to remove infected pigs rapidly and accurately (Álvarez et

177 al., 2019).

178 Immunology and nucleic acid detection are two main methods to diagnose ASF.

179 qPCR assays have many advantages compared with immunology methods, such as

180 simple operation processes, high sensitivity, high specificity and excellent repeatability. 
181 Thus, qPCR is the most commonly used laboratory test method to diagnose ASFV at 182 present, along with the ASFV antigen detection method recommended by the OIE (King

183 et al., 2003). However, it is difficult to determine CT values larger than 35 . The main

184 reason for the high CT values is the low viral content of the samples. The degradation

185 of nucleic acids due to improper preservation and transportation methods also results

186 in confusing data. The present study took advantage of a patented lymph node sampler

187 to puncture the skin in a minimally invasive manner to efficiently obtain lymph node

188 tissue. Moreover, an innovative nucleic acid protective fluid was used to prevent the

189 degradation of ASFV DNA. The qPCR data revealed that the ASFV content of the

190 lymph node samples was higher than that of other common kinds of samples, including

191 serum, oral fluid and nasal-rectal swabs, suggesting that the lymph node is an ideal

192 tissue for the diagnosis of ASF.

193 Practice has indicated that our synthetic solutions work well in the prevention and

194 control of ASF according to the contingency plan (Ministry-of-Agriculture-and-Rural-

195 Affairs, 2020). In general, we require all the pigs, materials and workers that intend to

196 enter the farm to be ASFV free by taking corresponding measures. Breeders should

197 inspect the herds daily and determine abnormal symptoms, including depression, fever,

198 inappetence, purple skin, blood spots, emesis, nosebleed, abortion, hematochezia and

199 death. The nasal swab, rectal swab and lymph node samples were collected immediately

200 and sent to a testing laboratory nearby. The laboratory was continuously running for

201 24-hour full-time service to give feedback to the decision makers within 8 hours. The 
202 positive pigs were removed with minimum contamination. The oral fluid and nasal-

203 rectal swab samples of the whole herd, as well as the whole divisional ground, were

204 collected at once. The positive pigs were removed again. The disinfection of the whole

205 farm was immediately executed using potent disinfectors. Seven days later, the next

206 cycle of census, elimination and disinfection were carried out until no positive results

207 occurred. Supported by these powerful biosecurity measures, lymph-node-sample-

208 based rapid and accurate definite diagnosis plays a dominant role in the systematic

209 projects.

210 In conclusion, this study took full advantage of the lymph node sampler to collect

211 lymph node samples from pigs in a minimally invasive way. The viral content of lymph

212 nodes, rather than serum or other swab samples of porcine secretions, was proven to be

213 the highest. The lymph-node-sampler-based sampling method helps to realize the

214 accurate diagnosis and elimination of ASF.

216 Declarations

217 Ethics approval and consent to participate

218 The authors confirm that all the procedures were conducted in adherence with the

219 the guidelines of New Hope Group Subcommittee of Experimental Animal Ethics

220 with no. of 151920.

\section{Consent for publication}

$222 \quad$ Not applicable. 
223

224

225

226

228

230

231

232

233

234

235

236

237

238

239

240 241 edited, and approved the manuscript.

\section{Availability of data and materials}

corresponding author on reasonable request.

\section{Competing interests}

\section{Funding}

(No. 31701424).

\section{Authors' contributions}

The dataset generated in this study is available from the first author and

The authors declare that there are no conflicts of interest.

This work was supported by the Integration and Demonstration of

Comprehensive Prevention and Control of ASFV by the Ministry of Science and

Technology (No. 2018YFC0840405), the New Hope Group Science and Technology

Leading 100-person Program Fund (NH201806), the Shandong Provincial Key

Laboratory Open Fund (SD2019BP102) and the China National Science Foundation

Study design and planning: Zhichun Yan, Xiaowen Li; animal experiments:

Wenchao Gao, Peng Yuan, Jingtao Li, Jincheng Yu, Weisheng Wu, Junxian Li;

virological work: Zhenwen Shao, Qiannan Yu, Yang Li; data processing and analysis:

Jing Ren, Mingyu Fan, Shiran Fan; drafting manuscript: Xiaowen Li, Yang Li;

critically revising manuscript prior to the submission: Zhichun Yan. All authors read,

\section{Acknowledgements}

243 The authors thank the conveniences from Shandong Provincial Laboratory of 
Swine Health Big Data and Intelligent Monitoring.

\section{References}

Álvarez, J., Bicout, D., Boklund, A., Bøtner, A., Depner, K., More, S.J., Roberts, H., Stahl, K., Thulke, H.H., Viltrop, A., Antoniou, S.E., Cortiñas Abrahantes, J., Dhollander, S., Gogin, A., Papanikolaou, A., Van der Stede, Y., González Villeta, L.C., Gortázar Schmidt, C., 2019. Research gap analysis on African swine fever. Efsa j 17, e05811.

Chapman, D.A., Darby, A.C., Da Silva, M., Upton, C., Radford, A.D., Dixon, L.K., 2011. Genomic analysis of highly virulent Georgia 2007/1 isolate of African swine fever virus. Emerg Infect Dis 17, 599-605.

Costard, S., Wieland, B., de Glanville, W., Jori, F., Rowlands, R., Vosloo, W., Roger, F., Pfeiffer, D.U., Dixon, L.K., 2009. African swine fever: how can global spread be prevented? Philos Trans R Soc Lond B Biol Sci 364, 2683-2696.

De Lorenzi, G., Borella, L., Alborali, G.L., Prodanov-Radulović, J., Štukelj, M., Bellini, S., 2020. African swine fever: A review of cleaning and disinfection procedures in commercial pig holdings. Research in veterinary science 132, 262-267.

Dixon, L.K., Chapman, D.A., Netherton, C.L., Upton, C., 2013. African swine fever virus replication and genomics. Virus Res 173, 3-14.

Gallardo, C., Fernandez-Pinero, J., Pelayo, V., Gazaev, I., Markowska-Daniel, I., Pridotkas, G., Nieto, R., Fernandez-Pacheco, P., Bokhan, S., Nevolko, O., Drozhzhe, Z., Perez, C., Soler, A., Kolvasov, D., Arias, M., 2014. Genetic variation among African swine fever genotype II viruses, eastern and central Europe. Emerg Infect Dis 20, 1544-1547.

Ge, S., Li, J., Fan, X., Liu, F., Li, L., Wang, Q., Ren, W., Bao, J., Liu, C., Wang, H., Liu, Y., Zhang, Y., Xu, T., Wu, X., Wang, Z., 2018. Molecular Characterization of African Swine Fever Virus, China, 2018. Emerg Infect Dis 24, 2131-2133.

Gogin, A., Gerasimov, V., Malogolovkin, A., Kolbasov, D., 2013. African swine fever in the North Caucasus region and the Russian Federation in years 2007-2012. Virus Res 173, 198-203.

Govoeyi, B., Agbokounou, A.M., Camara, Y., Ahounou, S.G., Dotche, I.O., Kiki, P.S., Abdou Karim, I.Y., Delabouglise, A., Antoine-Moussiaux, N., 2020. Social network analysis of practice adoption facing outbreaks of African Swine Fever. Prev Vet Med 179, 105008.

Guinat, C., Gubbins, S., Vergne, T., Gonzales, J.L., Dixon, L., Pfeiffer, D.U., 2016. Experimental pig-to-pig transmission dynamics for African swine fever virus, Georgia 2007/1 strain. Epidemiol Infect 144, 25-34.

Halasa, T., Botner, A., Mortensen, S., Christensen, H., Toft, N., Boklund, A., 2016. Control of African swine fever epidemics in industrialized swine populations. 
Vet Microbiol 197, 142-150.

Jackman, J.A., Hakobyan, A., Zakaryan, H., Elrod, C.C., 2020. Inhibition of African swine fever virus in liquid and feed by medium-chain fatty acids and glycerol monolaurate. J Anim Sci Biotechnol 11, 114.

King, D.P., Reid, S.M., Hutchings, G.H., Grierson, S.S., Wilkinson, P.J., Dixon, L.K., Bastos, A.D., Drew, T.W., 2003. Development of a TaqMan PCR assay with internal amplification control for the detection of African swine fever virus. Journal of virological methods 107, 53-61.

Li, X., Fan, S., Liu, C., Zhang, Z., Fan, M., 2020. A medical tissue sampler for livestock. CN211749759U.

Li, X., Tian, K., 2018. African swine fever in China. The Veterinary record 183, 300301.

Lichoti, J.K., Davies, J., Kitala, P.M., Githigia, S.M., Okoth, E., Maru, Y., Bukachi, S.A., Bishop, R.P., 2016. Social network analysis provides insights into African swine fever epidemiology. Prev Vet Med 126, 1-10.

Liu, J., Li, Z., Ren, X., Li, H., Lu, R., Zhang, Y., Ning, Z., 2019. Viral load and histological distribution of atypical porcine pestivirus in different tissues of naturally infected piglets. Arch Virol 164, 2519-2523.

Lu, G., Pan, J., Zhang, G., 2020. African swine fever virus in Asia: Its rapid spread and potential threat to unaffected countries. J Infect 80, 350-371.

Ma, J., Chen, H., Gao, X., Xiao, J., Wang, H., 2020. African swine fever emerging in China: Distribution characteristics and high-risk areas. Prev Vet Med 175, 104861.

Ministry-of-Agriculture-and-Rural-Affairs, 2020. African Swine Fever Response Implementation Plan. http://www.moa.gov.cn/nybgb/2020/202006/202007/t20200714_6348531.htm (accessed 20 April 2021).

Murphy, C., Bashiruddin, J.B., Quan, M., Zhang, Z., Alexandersen, S., 2010. Foot-andmouth disease viral loads in pigs in the early, acute stage of disease. The Veterinary record 166, 10-14.

Oganesyan, A.S., Petrova, O.N., Korennoy, F.I., Bardina, N.S., Gogin, A.E., Dudnikov, S.A., 2013. African swine fever in the Russian Federation: spatio-temporal analysis and epidemiological overview. Virus Res 173, 204-211.

Olesen, A.S., Lohse, L., Boklund, A., Halasa, T., Belsham, G.J., Rasmussen, T.B., Bøtner, A., 2018. Short time window for transmissibility of African swine fever virus from a contaminated environment. Transbound Emerg Dis 65, 1024-1032.

Olesen, A.S., Lohse, L., Boklund, A., Halasa, T., Gallardo, C., Pejsak, Z., Belsham, G.J., Rasmussen, T.B., Botner, A., 2017. Transmission of African swine fever virus from infected pigs by direct contact and aerosol routes. Vet Microbiol 211, 92102.

Palya, V., Homonnay, Z.G., Mato, T., Kiss, I., 2018. Characterization of a PCV2d-2 isolate by experimental infection of pigs. Virol J 15, 185. 
Penrith, M.L., Vosloo, W., 2009. Review of African swine fever: transmission, spread and control. J S Afr Vet Assoc 80, 58-62.

Ren, J., Li, X., Fan, M., Wang, J., Yu, J., Gao, W., 2020. An oral fluid virus DNA protector and its preparation method and application. CN202010716933.6.

Senthilkumar, D., Rajukumar, K., Sen, A., Kumar, M., Shrivastava, D., Kalaiyarasu, S., Gautam, S., Singh, F., Kulkarni, D.D., Singh, V.P., 2018. Pathogenic characterization of porcine reproductive and respiratory syndrome virus of Indian origin in experimentally infected piglets. Transbound Emerg Dis 65, 1522-1536.

Tulman, E.R., Delhon, G.A., Ku, B.K., Rock, D.L., 2009. African swine fever virus. Curr Top Microbiol Immunol 328, 43-87.

Wilkinson, P.J., Donaldson, A.I., 1977. Transmission studies with African swine fever virus. The early distribution of virus in pigs infected by airborne virus. J Comp Pathol 87, 497-501.

Yu, G., Hatta, A., Periyannan, S., Lagudah, E., Wulff, B.B.H., 2017. Isolation of Wheat Genomic DNA for Gene Mapping and Cloning. Methods in molecular biology 1659, 207-213.

Zhao, D., Liu, R., Zhang, X., Li, F., Wang, J., Zhang, J., Liu, X., Wang, L., Zhang, J., Wu, X., Guan, Y., Chen, W., Wang, X., He, X., Bu, Z., 2019. Replication and virulence in pigs of the first African swine fever virus isolated in China. Emerg Microbes Infect 8, 438-447.

Zhou, X., Li, N., Luo, Y., Liu, Y., Miao, F., Chen, T., Zhang, S., Cao, P., Li, X., Tian, K., Qiu, H.J., Hu, R., 2018. Emergence of African Swine Fever in China, 2018. Transbound Emerg Dis 65, 1482-1484.

\section{Figure legends}

Figure 1. The structure of inguinal lymph node sampler. a The appearance of the assembled lymph node sampler. b Component disassembly of the lymph node

sampler. $\mathbf{c}$ The barb of needle. $\mathbf{d}$ The connection rod can be inserted into the needle to

extrude the tissue.

Figure 2. Three steps to obtain inguinal lymph node samples using a sampler. a

Find and pinch the inguinal lymph node. b Puncture the skin and tissue. $\mathrm{c}$ Pull out the 
358 sampler and press the handle several times to squeeze the tissue out. $d$ Enlarged view

359 of squeezed lymph node tissue.

$361 \quad$ Figure 3. The ASFV content of lymph nodes and other kinds of collected

362 samples. Eight kinds of porcine samples were collected, and their ASFV content was

363 detected by qPCR. Individual CT values are presented.

365 Figure 4. A larger proportion of strongly positive results was found in lymph

366 node samples. The CT values were sorted into different ranges. The distribution of

367 each 5 CT ranges is shown in the figure. Values smaller than 30 were identified as

368 strongly positive results.

Figure 5. ASFV-positive pigs were removed within 3 weeks according to the

371 viral content of lymph nodes. The ASFV-positive farms judged the suspected pigs by

372 the viral content of the lymph nodes. The positive pigs were removed from the herd,

373 and the surroundings were disinfected thoroughly by irrigation with $3 \%$ sodium

374 hypochlorite until ASFV nucleic acid was undetectable. The results of nucleic acid

375 detection are shown. Red: positivity; green: negativity. 


\section{Figures}
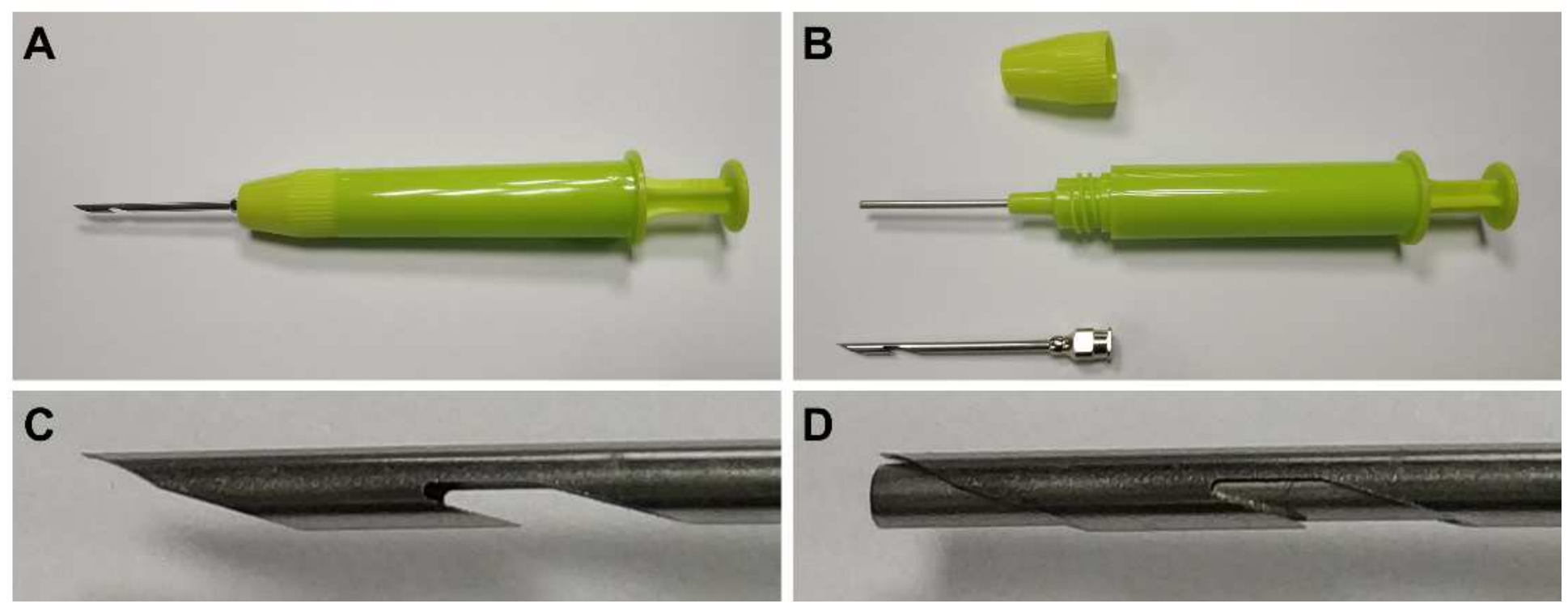

\section{Figure 1}

The structure of inguinal lymph node sampler. a The appearance of the assembled lymph node sampler. b Component disassembly of the lymph node sampler. c The barb of needle. $d$ The connection rod can be inserted into the needle to extrude the tissue. 


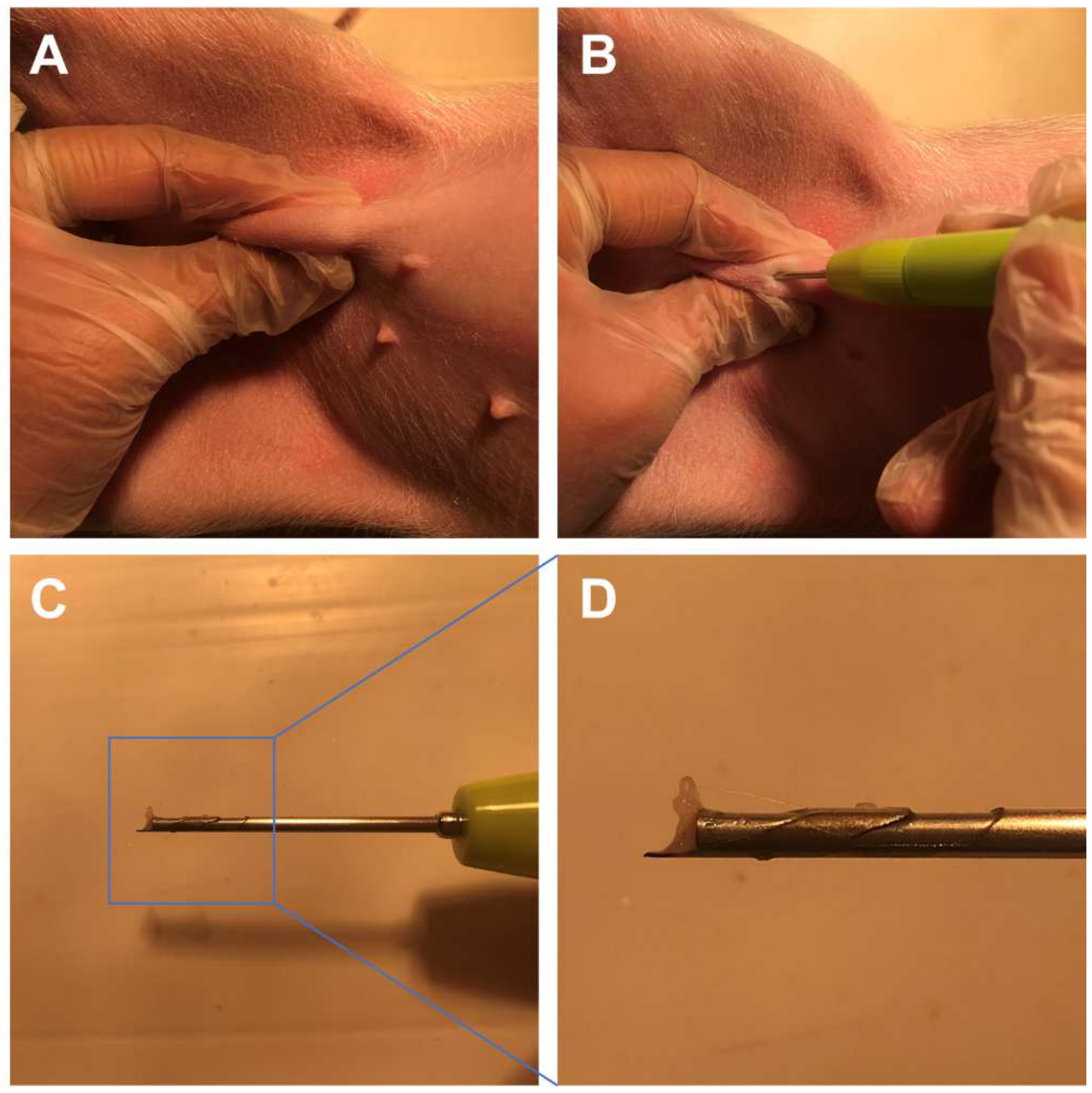

Figure 2

hree steps to obtain inguinal lymph node samples using a sampler. a Find and pinch the inguinal lymph node. $b$ Puncture the skin and tissue. $c$ Pull out the sampler and press the handle several times to squeeze the tissue out. $d$ Enlarged view of squeezed lymph node tissue. 


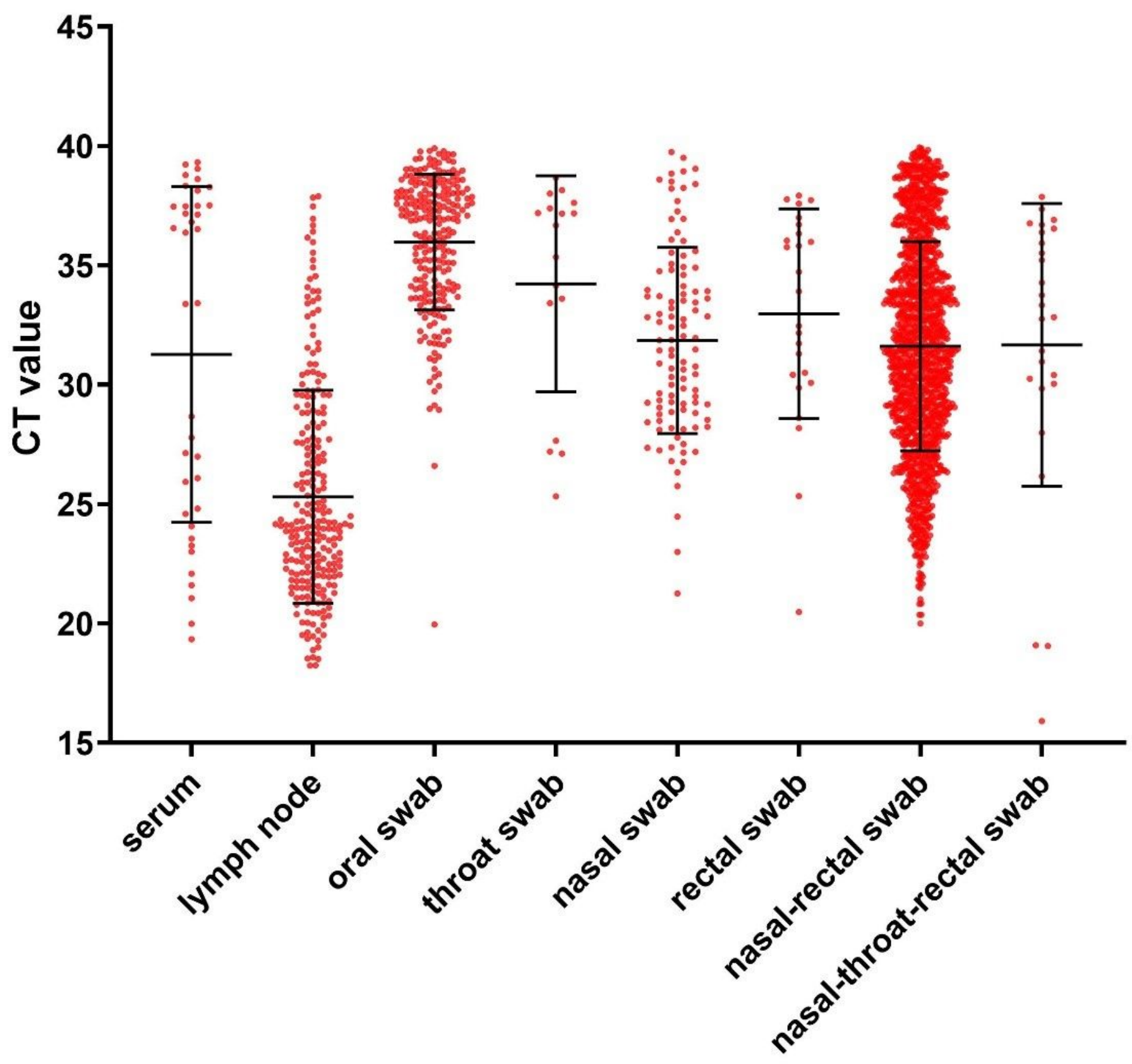

Figure 3

The ASFV content of lymph nodes and other kinds of collected samples. Eight kinds of porcine samples were collected, and their ASFV content was detected by GPCR. Individual CT values are presented. 


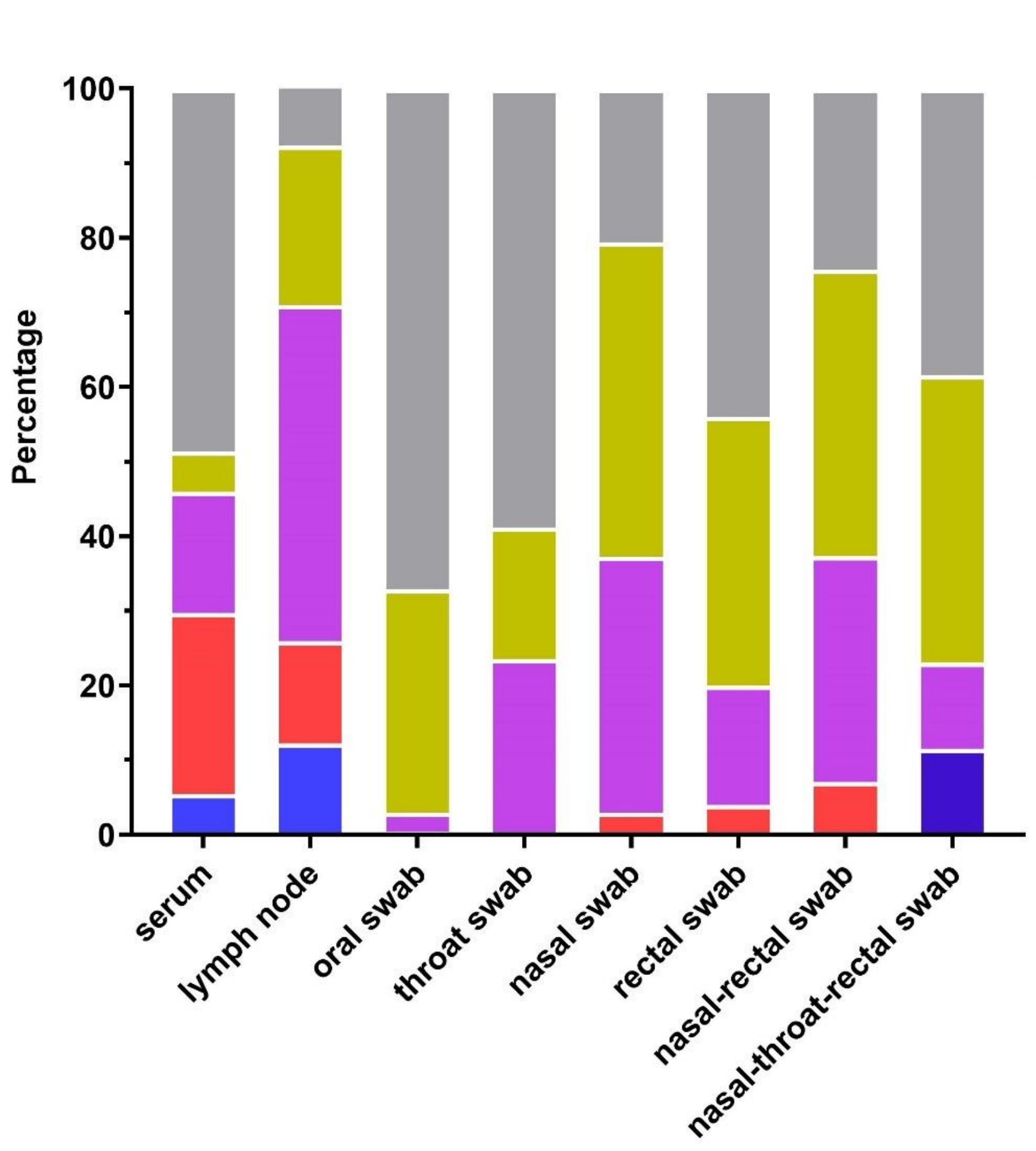

Figure 4

A larger proportion of strongly positive results was found in lymph node samples. The CT values were sorted into different ranges. The distribution of each $5 \mathrm{CT}$ ranges is shown in the figure. Values smaller than 30 were identified as strongly positive results. 


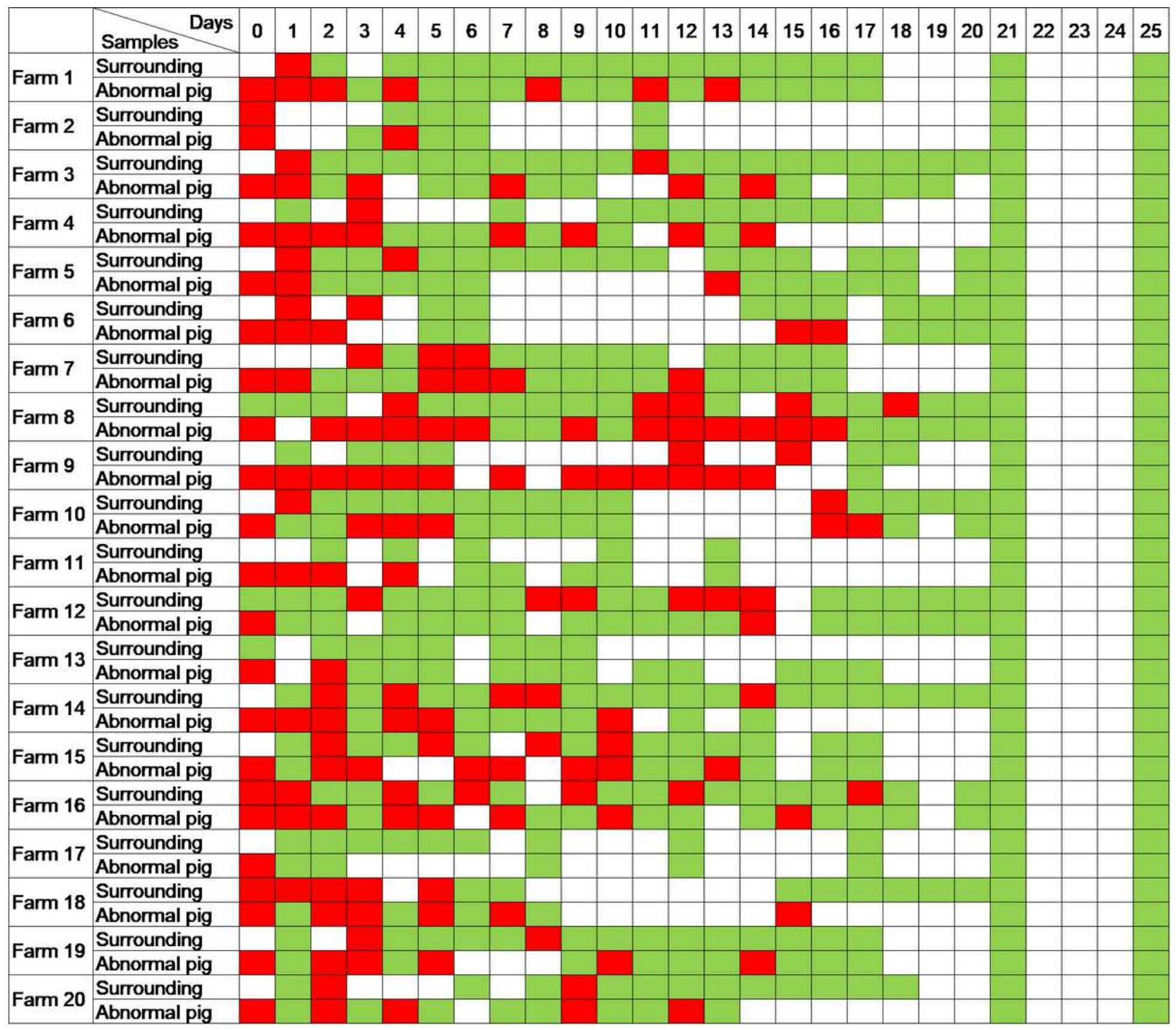

Figure 5

ASFV-positive pigs were removed within 3 weeks according to the viral content of lymph nodes. The ASFV-positive farms judged the suspected pigs by the viral content of the lymph nodes. The positive pigs were removed from the herd, and the surroundings were disinfected thoroughly by irrigation with $3 \%$ sodium hypochlorite until ASFV nucleic acid was undetectable. The results of nucleic acid detection are shown. Red: positivity; green: negativity. 